\title{
First demonstration of the echo-enabled harmonic generation technique for short-wavelength seeded free electron lasers
}

\author{
D. Xiang, E. Colby, M. Dunning, S. Gilevich, C. Hast, K. Jobe, D. McCormick, J. Nelson, \\ T.O. Raubenheimer, K. Soong, G. Stupakov, Z. Szalata, D. Walz, S. Weathersby, M. Woodley \\ SLAC National Accelerator Laboratory, Menlo Park, CA, 94025, USA \\ P-L. Pernet \\ École Polytechnique Fédérale de Lausanne, Lausanne, Switzerland
}

(Dated: July 17, 2010)

\begin{abstract}
We report the first experimental demonstration of the echo-enabled harmonic generation (EEHG) technique which holds great promise for generation of high power, fully coherent short-wavelength radiation. In this experiment, coherent radiation at the 3rd and 4th harmonic of the second seed laser is generated from the so-called beam echo effect. The experiment confirms the physics behind this technique and paves the way for applying the EEHG technique for seeded x-ray free electron lasers.

PACS numbers: $41.60 . \mathrm{Cr}$
\end{abstract}

Free electron lasers (FELs) can provide high-intensity coherent short-wavelength radiation which is essential for the studies of molecular and atomic dynamics. In the xray wavelength, the two leading concepts are the selfamplified spontaneous emission (SASE) configuration $[1,2]$ and the high-gain harmonic generation (HGHG) scheme [3, 4]. One FEL working in the SASE mode has been successfully operated at hard x-ray wavelengths [5]. While the radiation from a SASE FEL has excellent transverse coherence, it typically has rather limited temporal coherence because a SASE FEL starts from electron beam shot noise. Alternatively, the HGHG scheme allows the generation of fully coherent radiation by upconverting the frequency of a high-power seed laser. However, due to the relatively low up-frequency conversion efficiency, multiple stages of HGHG FELs are needed [6] in order to generate coherent x-rays from a UV laser.

The up-frequency conversion efficiency can be greatly improved with the recently proposed echo-enabled harmonic generation (EEHG) technique [7, 8]. In the EEHG scheme, the beam is energy modulated by a laser with wave number $k_{1}$ in the first modulator and then sent through a chicane with strong dispersion after which the modulation obtained in the first modulator is macroscopically smeared. Simultaneously, complicated fine structures are introduced into the phase space of the beam. A second laser with wave number $k_{2}$ is used to further modulate the beam energy in the second modulator. After passing through a second chicane the echo signal then occurs at the wave number $k_{E}=n k_{1}+m k_{2}$ as a recoherence effect, where $n$ and $m$ are integers. The main advantage of EEHG is that the bunching factor is a very slowly decaying function of the harmonic number, thus allowing the generation of coherent soft x-ray radiation directly from a UV seed laser in a single stage.

The remarkable up-frequency conversion efficiency of the EEHG technique has stimulated world-wide interest in using EEHG to achieve fully coherent radiation in the x-ray wavelength from UV seed lasers [9-11]. While sig- nificantly relaxing the requirements on laser power and beam slice energy spread as compared to the HGHG scheme, EEHG requires more challenging control of the beam dynamics as the beam goes through the undulators and chicanes, because it involves a long-term memory of the beam phase space correlations.

In this Letter we report the first experimental demonstration of the EEHG technique at the Next Linear Collider Test Accelerator (NLCTA) at SLAC. The experiment at NLCTA [12] is a generic EEHG proof-of-principle experiment that uses two different lasers (the first laser wavelength is $\lambda_{1}=795 \mathrm{~nm}$ and the second is $\lambda_{2}=1590$ $\mathrm{nm}$ ) in the modulators. The echo signals generated by the interplay of the two lasers at the 3rd and 4th harmonic of the second laser were observed and clearly differentiated from those generated by the lasers individually. The dependence of the echo signal wavelength as a function of beam energy chirp was measured and found to be in good agreement with theory. The experiment verifies the EEHG theory and indicates that scaling to shortwavelength echo-seeded x-ray FELs may be possible.

The design considerations, commissioning and the experimental details at the NLCTA are discussed in $[13,14]$. The layout of the experiment is schematically shown in Fig. 1. The experiment is based on the existing NLCTA facilities where the electron beam is generated in a 1.6 cell S-band photocathode rf gun with a 0.5 ps frequencytripled $(266 \mathrm{~nm})$ Ti:Sapphire laser and is boosted to 60 $\mathrm{MeV}$ in an $\mathrm{x}$-band linac (X1). A flexible chicane (C-1) which can provide either positive or negative momentum compaction is available to manipulate the beam's longitudinal phase space. In this experiment it is set to provide a positive momentum compaction of $R_{56}=76 \mathrm{~mm}$. The facility can provide $20 \sim 40 \mathrm{pC}$ electron bunches with a normalized emittance of about $8 \mathrm{~mm} \cdot \mathrm{mrad}$.

In order to conduct the EEHG experiment in the NLCTA tunnel, another x-band structure (X2) was added downstream of $\mathrm{C}-1$ to further accelerate the beam to $120 \mathrm{MeV}$. The main components of the EEHG beam 
line consist of 3 chicanes ( $\mathrm{C} 0, \mathrm{C} 1$ and $\mathrm{C} 2$ ), 3 undulators (U1, U2 and U3), several insertable optical transition radiation (OTR) screens for electron and laser beam position and size measurements, and quadrupoles for beam matching and focusing.

The mini-chicane $(\mathrm{C} 0)$ is used to generate an orbit bump to allow laser injection into the first undulator (U1). The beam is modulated by the $795 \mathrm{~nm}$ laser (1 ps FWHM, Ti:Sapphire laser) in U1 (10 periods with a period of $3.3 \mathrm{~cm}$ and a $K$ value of 1.82$)$. After passing through $\mathrm{C} 1$ complicated fine structures are introduced into the beam longitudinal phase space. The beam is again modulated by the $1590 \mathrm{~nm}$ laser (0.7 ps FWHM, produced by an optical parametric amplifier system pumped with the $795 \mathrm{~nm}$ laser) in the second undulator U2 (10 periods with a period of $5.5 \mathrm{~cm}$ and a $K$ value of 2.09). Finally density modulations at shorter wavelength are generated after passage through $\mathrm{C} 2$. The density-modulated beam will produce coherent radiation in U3 (10 periods with a period length of $2 \mathrm{~cm}$ ) which is presently tuned to $318 \mathrm{~nm}$. The radiation generated in $\mathrm{U} 3$ is reflected by a downstream OTR screen to a spectrometer. The undulators $\mathrm{U} 1$ and $\mathrm{U} 2$ were built by the STI Optronics company and U3 was built by LBNL.

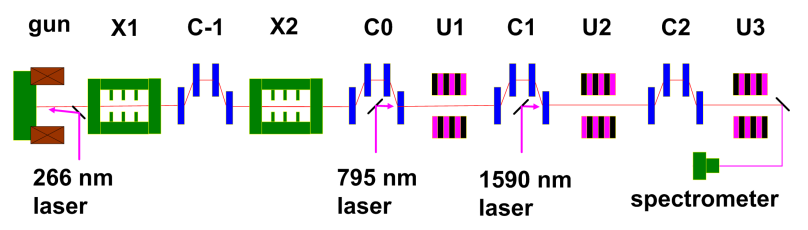

FIG. 1: Schematic of the EEHG experiment at SLAC

The beam-laser interaction is achieved when electron and laser beam overlap both spatially and temporally in the modulators. The spatial overlap is achieved by steering the laser to the same position as the beam on the OTR screens upstream and downstream of the undulators. In the experiment the laser size is about two times larger than that of the electron beam [14] to provide uniform modulation in transverse direction. An OTR screen immediately downstream of each undulator can be inserted to reflect out the laser and undulator radiation for temporal synchronization. The radiation is detected by a fast photodiode ( $2 \mathrm{GHz}$ bandwidth) and is analyzed with an oscilloscope. By referencing the signals to an external trigger, the laser and beam can be synchronized to within approximately $30 \mathrm{ps}$. More precise timing is done by using a scanning delay stage and measuring the coherent radiation enhancement that is produced when the beam is energy modulated by each modulator and further bunched by the subsequent dispersive section [14].

After setting the delay stages such that the two lasers interact with the electron beam simultaneously, the EEHG experiment was set up to generate the 3rd and 4th harmonics of the second laser. The beam was accelerated about 10 degrees off crest in X1 so that a positive energy chirp is imparted in X1 and the beam is decompressed to about 2.5 ps rms after $\mathrm{C}-1$. The momentum compaction of chicane $\mathrm{C} 1$ was set to $R_{56}^{(1)}=4.9 \mathrm{~mm}$ and that of chicane $\mathrm{C} 2$ was set to $R_{56}^{(2)}=2.8 \mathrm{~mm}$. The rf phase of X2 was adjusted to cancel the energy chirp imparted in X1 so that the beam had a minimal global energy spread at the entrance to $\mathrm{C} 0$. This also minimized the energy chirp in the beam. The radiation generated in $\mathrm{U} 3$ is reflected out by the OTR screen and guided into a spectrometer which consists of a 300 lines/mm grating, an optical lens and a CCD detector. The wavelength calibration in the measurement is achieved using the incoherent undulator radiation which has a continuous spectrum, together with two bandpass filters (one at $395 \mathrm{~nm}$ and the other at 531 $\mathrm{nm}$; both have a bandwidth of $11 \mathrm{~nm}$ ). With this setup the radiation wavelength from $350 \mathrm{~nm}$ to about $600 \mathrm{~nm}$ can be measured in a single shot. While the resolving power of the grating is about 1-2 $\mathrm{nm}$, the relatively large source size limits the resolution of our measurement system to about $4 \mathrm{~nm}$.

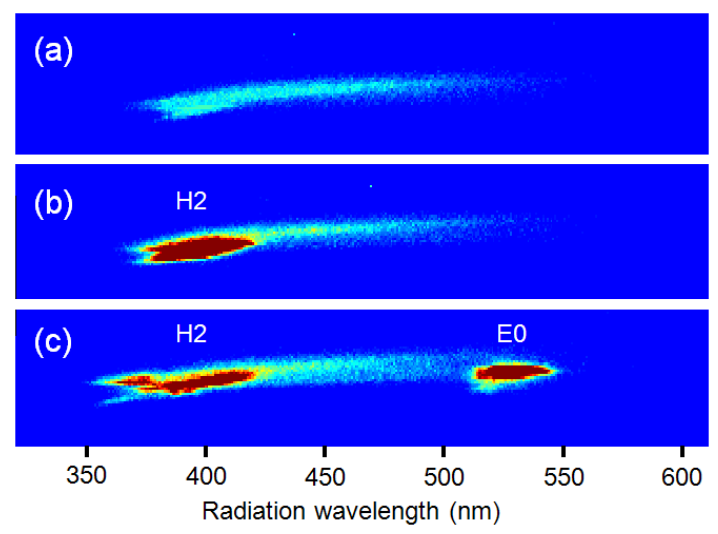

FIG. 2: Spectrum of the radiation at the exit of U3 when beam energy chirp is minimized:(a) only $1590 \mathrm{~nm}$ laser is on; (b) only $795 \mathrm{~nm}$ laser is on; (c) both lasers are on.

Results are shown in Fig. 2 when the beam energy chirp is minimized. The spectrum of the coherent radiation is broadened in our experiment due to the relatively large residual chirp from the X-band linac. Henceforth, only the center wavelength of the coherent radiation is quoted. In the experiment the $1590 \mathrm{~nm}$ laser was first turned on and its 3rd and 4th harmonics were observed (not shown in Fig. 2) at the CCD when the laser power is high enough to generate an energy modulation amplitude much larger than the beam slice energy spread. The $1590 \mathrm{~nm}$ laser power was then reduced until its 3rd and 4th harmonics disappeared. The measured radiation spectrum is shown in Fig. 2a. Then the $795 \mathrm{~nm}$ laser was also turned on and its power was set to provide a peak energy modulation of about $20-30 \mathrm{keV}$ in U1. After finely adjusting the $795 \mathrm{~nm}$ laser timing, the 3rd harmonic of the $1590 \mathrm{~nm}$ laser with a center wavelength at about $530 \mathrm{~nm}$ (E0 in Fig. 2c) was observed (see Fig. 2c). Note the radiation at $530 \mathrm{~nm}$ can not be generated by the $795 \mathrm{~nm}$ laser alone, because it is not its harmonic. It is not generated by 
the $1590 \mathrm{~nm}$ laser alone either, because the laser power is significantly reduced to provide a very small energy modulation. Therefore, the radiation E0 at about 530 $\mathrm{nm}$ when both lasers are turned on is believed to be the echo signal generated by the interplay of the two lasers with the set $n=-1, m=5$. To further confirm this, the $1590 \mathrm{~nm}$ laser was turned off, and the measured radiation spectrum in Fig. 2b shows only the wavelength around $400 \mathrm{~nm}$ (H2), which is the 2nd harmonic of the $795 \mathrm{~nm}$ laser, is enhanced.

It should be pointed out that an echo signal $(n=$ $-1, m=6$ ) at about $400 \mathrm{~nm}$ might be generated in this setup as well. Since it has the same wavelength as the H2 signal, they both appear at the same position on the CCD and are difficult to separate. In order to distinguish them, we repeated the experiment with an energychirped beam. Analysis shows that with sufficient energy chirp in the beam, the echo signal and that generated by the lasers individually will shift to different wavelengths and may be separated with the spectrometer.

It has been shown in [15] that with an energy chirp in the beam, the harmonic radiation wavelength generated by a single laser will shift to $\lambda=\lambda_{0} / C$, where $\lambda_{0}$ is the radiation wavelength when the beam has a vanishing energy chirp, $C=1 /\left(1+h R_{56}\right)$ is the compression factor of the chicane and $h=d \delta / d z$ is the energy chirp factor at the entrance to the chicane. The EEHG theory predicts a quite different wavelength dependence [16]. With an energy chirp $h$, the wave number of the echo signal will shift to,

$$
k_{E}(h)=\frac{n k_{1}+\left(1+h R_{56}^{(1)}\right) m k_{2}}{1+h\left(R_{56}^{(1)}+R_{56}^{(2)}\right)} .
$$

To verify this prediction, we adjusted the phase of X2 to imprint considerable positive energy chirp on the electron beam. As shown in Fig. 3a, due to the energy chirp and the bunch decompression in $\mathrm{C} 2$, the 4th harmonic of the $1590 \mathrm{~nm}$ laser (H4) shifted from $397.5 \mathrm{~nm}$ to about $430 \mathrm{~nm}$, and the 3rd harmonic of the $1590 \mathrm{~nm}$ laser (H3) shifted from $530 \mathrm{~nm}$ to about $570 \mathrm{~nm}$. Here the $1590 \mathrm{~nm}$ laser power is increased to provide an energy modulation amplitude of about $10 \mathrm{keV}$ in the beam so that the harmonic radiation generated by the $1590 \mathrm{~nm}$ laser alone can be clearly seen. The radiation is measured downstream of U3, so the compression factor for the modulation generated in $\mathrm{U} 2$ is $C_{U 2}=\left(1+h R_{56}^{(1)}\right) /\left(1+h\left(R_{56}^{(1)}+R_{56}^{(2)}\right)\right)$, where $h$ is the chirp at the entrance to chicane C1. The compression factor is $C_{U 2}=0.9244$ and the chirp is inferred to be $h=33.4 \mathrm{~m}^{-1}$.

The modulation generated in U1 will be decompressed in both $\mathrm{C} 1$ and $\mathrm{C} 2$, and the corresponding compression factor is $C_{U 1}=1 /\left(1+h\left(R_{56}^{(1)}+R_{56}^{(2)}\right)\right)$. With this chirp the compression factor for the modulation induced by the $795 \mathrm{~nm}$ laser is found to be $C_{U 1}=0.7937$. Accordingly, the wavelength for the $2 \mathrm{nd}$ harmonic of the $795 \mathrm{~nm}$ laser (H2) should shift from $397.5 \mathrm{~nm}$ to $501 \mathrm{~nm}$ while it was measured at about $499 \mathrm{~nm}$, which is well within the
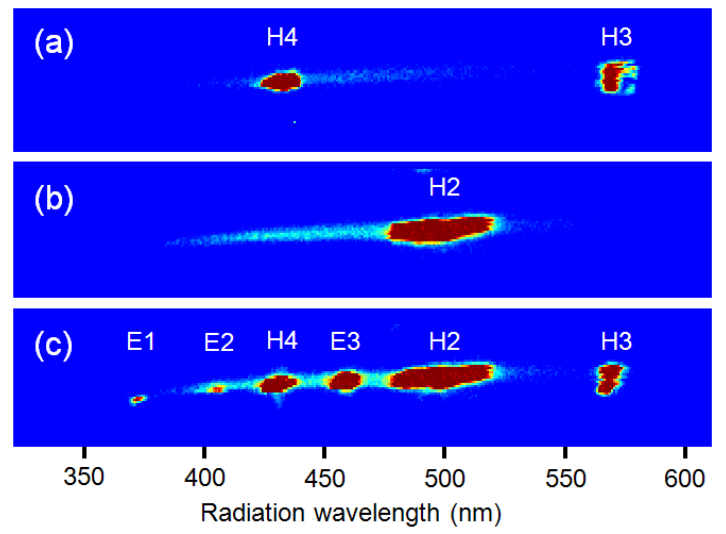

FIG. 3: Spectrum of the radiation at the exit of U3 when beam has considerable energy chirp:(a) only $1590 \mathrm{~nm}$ laser is on; (b) only $795 \mathrm{~nm}$ laser is on; (c) both lasers are on.

experimental resolution (Fig. 3b).

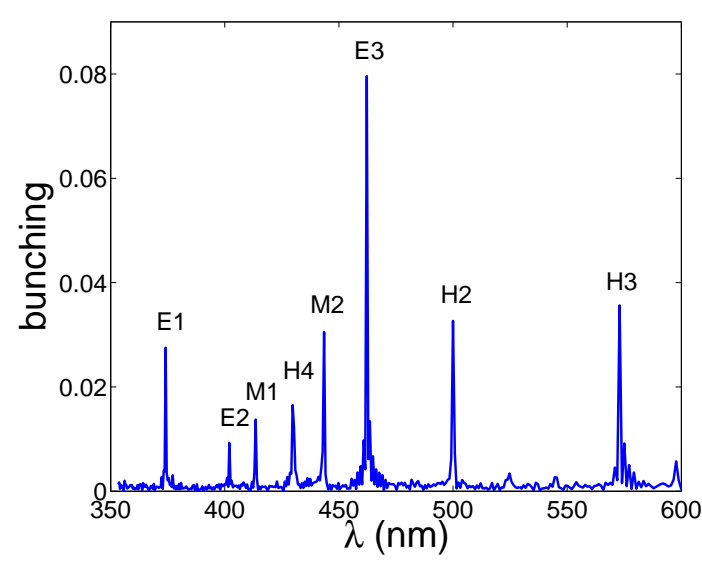

FIG. 4: Bunching at various wavelengths from simulation.

When both lasers are turned on, 3 additional echo signals with different wavelengths E1, E2 and E3 are observed (Fig. 3c). The simulated bunching factors for various wavelengths with this chirp are shown in Fig. 4. The beam typically has a slice energy spread of about $1 \mathrm{keV}$ and was decompressed by about a factor of 4 in chicane C-1. Consequently, a slice energy spread of $0.25 \mathrm{keV}$ is assumed in the simulation. The energy modulation amplitudes in U1 and U2 are assumed to be 21 and 10 $\mathrm{keV}$, which is consistent with the experimental values. With these specific parameters, simulation predicts that enhanced coherent radiation at 8 wavelengths may be generated in our experiment.

Analysis shows that the echo signal E1 in Fig. 3 and Fig. 4 is generated with the set $n=-5, m=4$. It follows from Eq. (1) that with this chirp the wavelength of E1 should shift from $397.5 \mathrm{~nm}$ to $374 \mathrm{~nm}$ and experimentally it was shifted to about $373 \mathrm{~nm}$. Similarly the echo signal E2 is found to be generated with the set $n=-1, m=6$. The wavelength of E2 should shift from $397.5 \mathrm{~nm}$ to 402 
$\mathrm{nm}$ and was measured at $405 \mathrm{~nm}$. E3 is generated with the set $n=1, m=2$. The wavelength of E3 should shift from $530 \mathrm{~nm}$ to $462 \mathrm{~nm}$ and was measured at about 458 $\mathrm{nm}$. Considering the resolution of the spectromter and some uncertainties of the beam chirp, the experimental results are in good agreement with the EEHG theory.

It should be pointed out that the simulated signals M1 (414 nm) and M2 (444 nm), are however, missing in our experiment. From simulation we found that the strength of the spectral lines are sensitive functions of modulation amplitudes, which are not well known in the experiment; this may account for the discrepancy. Nevertheless, all the observed spectral lines in the experiment can be well explained with the EEHG theory.

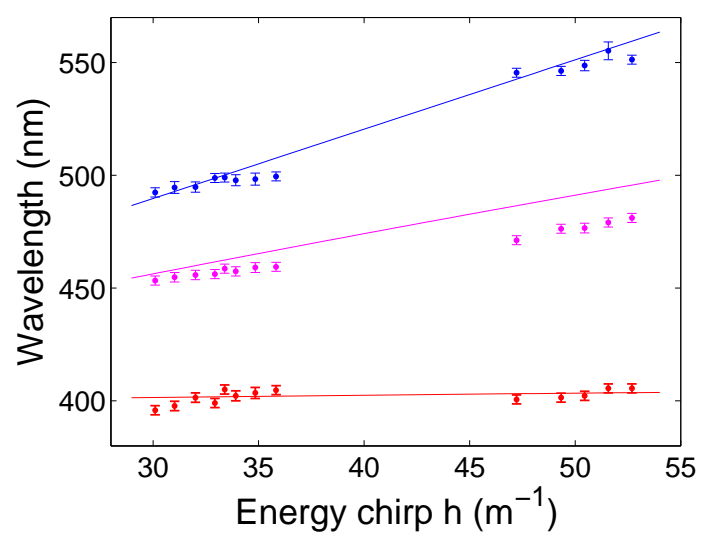

FIG. 5: Radiation wavelength vs beam energy chirp for the echo signal E2 (red), E3 (magenta) and that from the $795 \mathrm{~nm}$ laser alone $\mathrm{H} 2$ (blue).

To further benchmark the EEHG theory, the beam energy chirp is varied and the wavelengths of the harmonic radiation for $\mathrm{E} 2, \mathrm{E} 3, \mathrm{H} 2$ and $\mathrm{H} 4$ are measured and shown in Fig. 5 (the E1 signal, being close to the cut-off wavelength of the diagnostic system, was not observed in every shot, and is not included in Fig. 5). The chirp factor is inferred from the wavelength shift of $\mathrm{H} 4$. The blue dots are the measured wavelength for the 2nd harmonic of the $795 \mathrm{~nm}$ laser (H2) and the blue line is the theoretical prediction. The red and magenta dots are the measured wavelength for the echo signal E2 and E3, and the red and magenta lines are the prediction in Eq. (1). The experimental results are in good agreement with the theory. The results confirm that the harmonic radiation wavelength generated by a single laser scales as the compression factor and the sensitivity of the echo signal to the beam chirp depends on $n$ and $m$. Specifically the echo signal generated with the set $n=-1, m=6$ is insensitive to the beam chirp.

It is worth mentioning that echo signals at even shorter wavelength could be generated in this experiment setup as well. However, the current diagnostics limit the measurement to optical wavelengths because the transmission of the spectrometer lens drops rapidly below 380 nm. Currently the spectrometer is being upgraded with a UV grade lens to explore the EEHG technique in the shorter wavelength range.

In summary, we presented the first experimental demonstration of the EEHG technique which allows far higher harmonic numbers to be accessed as compared to the normal limit arising from the beam slice energy spread. The good agreement between the EEHG theory and our experimental results confirms the physics behind this technique and paves the way for applying the EEHG technique for future seeded x-ray FELs.

We thank C. Adolphsen, K. Bane, J. Byrd, A. Chao, J. Corlett, J. Cruz, Y. Ding, P. Emma, W. Fawley, J. Frederico, J. Frisch, G. Hays, Z. Huang, C. Hudspeth, H. Loos, H.-D. Nuhn, G. Penn, S. Prestemon, J. Qiang, M. Racine, R. Schlueter, M. Venturini, W. Wan, F. Wang, X.-J. Wang, W. White, J. Wu and A. Zholents for helpful discussions, comments and commissioning assistance. This work was supported by the US DOE Office of Basic Energy Sciences using the NLCTA facility which is partly supported by US DOE Office of High Energy Physics under Contract No. DE-AC02-76SF00515.
[1] A. Kondratenko and E. Saldin, Part. Accel. 10, 207 (1980).

[2] R. Bonifacio, C. Pellegrini, and L.M. Narducci, Opt. Commun. 50, 373, (1984).

[3] L.-H. Yu, Phys. Rev. A, 44, 5178 (1991).

[4] L.-H. Yu, et al., Science, 289, 932 (2000).

[5] P. Emma, et al., Nature Photon., to be published, (2010).

[6] J. Wu and L. H. Yu, Nucl. Instrum. Methods Phys. Res., Sect. A 475, 104 (2001).

[7] G. Stupakov, Phys. Rev. Lett, 102, 074801 (2010).

[8] D. Xiang and G. Stupakov, Phys. Rev. ST-AB, 12, 030702 (2009).

[9] D. Xiang and G. Stupakov, in Proceedings of PAC 09 (IEEE, Vancouver, 2009).
[10] E. Allaria, et al., in Proceedings of FEL 09 (Liverpool, 2009), p.39.

[11] S. Reiche et al., in Proceedings of FEL 09 (Liverpool, 2009), p.51.

[12] "NLC test accelerator conceptual design report", SLACReport-411, (1993).

[13] M. Dunning et al., in Proceedings of IPAC 10 (IEEE, Kyoto, 2010), p.2293.

[14] D. Xiang et al., in Proceedings of IPAC 10 (IEEE, Kyoto, 2010), p.2299.

[15] T. Shaftan and L.-H. Yu, Phys. Rev. E 71, 046501 (2005).

[16] Z. Huang et al., in Proceedings of FEL 09 (Liverpool, 2009), p.127. 